\title{
Assessment of Doppler velocimetry versus nonstress test in antepartum surveillance of high risk pregnancy
}

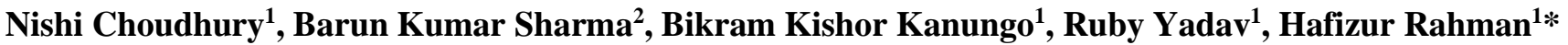

\author{
${ }^{1}$ Department of Obstetrics and Gynecology, Sikkim Manipal Institute of Medical Sciences, Gangtok, Sikkim, India \\ ${ }^{2}$ Department of Radiodiagnosis, Sikkim Manipal Institute of Medical Sciences, Gangtok, Sikkim, India
}

Received: 10 December 2016

Accepted: 05 January 2017

\author{
*Correspondence: \\ Dr. Hafizur Rahman, \\ E-mail: hafizezzy@gmail.com
}

Copyright: () the author(s), publisher and licensee Medip Academy. This is an open-access article distributed under the terms of the Creative Commons Attribution Non-Commercial License, which permits unrestricted non-commercial use, distribution, and reproduction in any medium, provided the original work is properly cited.

\begin{abstract}
Background: High risk pregnancies increase the maternal and fetal morbidity and mortality; and there is a need for appropriate investigation which can diagnose it early and predicts the morbidity and mortality. The objectives of this study were to compare the efficacy of Doppler velocimetry studies and NST in predicting fetal compromise in utero and compare their ability in predicting the perinatal outcome in cases of high risk pregnancies.

Methods: It was a prospective cross-sectional hospital based study conducted at Central Referral Hospital (CRH) which is a teaching hospital of Sikkim Manipal Institute of Medical Sciences (SMIMS). The study was conducted over a period of eighteen months between November 2012 and April 2014. One hundred consecutive cases of high risk pregnancies were enrolled into the study and investigated with NST (non-stress test) and Doppler velocimetry and results were correlated with perinatal outcome. In all cases, accurate gestational age was established from detailed menstrual history and ultrasonographic evidence of gestational age. Detailed examination, history and investigation were undertaken in each patient. Inclusion criteria were patients with singleton pregnancy beyond 34 weeks having one or more high risk factors. In these patients antenatal surveillance was done by Doppler and NST and results of these tests were correlated with perinatal outcome. Based on the Doppler velocimetry and NST results, the study population was divided in to four groups. Pregnant women below 34 weeks, multiple pregnancy and women with no risk factors were excluded from the study.

Results: Maximum (63\%) patients belonged to pregnancy induced hypertension (PIH) group, followed by oligohydramnios (11\%), and gestational diabetes mellitus (GDM). The study showed that patients with both NST and Doppler waveform abnormal (group D) had the highest percentage of neonatal complication, NICU admissions and perinatal deaths. Even those patients with NST normal but Doppler velocimetry abnormal (group B) had comparatively higher neonatal complications. However, in group with NST abnormal and Doppler velocimetry normal (group C) had no fetal compromise. It was observed that normal NST and normal Doppler velocimetry were not statistically different in predicting fetal compromise and prediction value was low. But abnormal Doppler had statistically significant $(\mathrm{p}$ value $=0.021$ ) predictive value in detecting fetal compromise. In cases with abnormal Doppler and fetal compromise, NST was still normal showing that abnormal Doppler waveform was better in predicting the bad perinatal outcome. Three out of 100 cases had absent end diastolic flow (AEDF) and all 3 were associated with perinatal morbidity with 2 perinatal deaths. Cerebroplacental ratio was $<1.08$ in seven cases and all seven had neonatal complications including 3 neonatal deaths which also had AEDF. Thus, cerebroplacental ratio was better in detecting fetal compromise as compared to NST.

Conclusions: Doppler velocimetry was better in predicting fetal compromise in comparison to NST in high risk pregnancies. Normal NST and normal Doppler velocimetry were not significantly different in prediction of fetal outcome. Abnormal Doppler value was better in predicting fetal compromise in comparison to abnormal NST.
\end{abstract}

Keywords: Doppler velocimetry, High risk pregnancy, Nonstress test, Perinatal outcome 


\section{INTRODUCTION}

A high-risk pregnancy is one that threatens the health or life of the mother or her fetus. Antepartum foetal surveillance is of immense importance for detection of fetal compromise in utero in high risk pregnancies like gestational hypertension, diabetes mellitus, fetal growth restriction, post-dated pregnancy. Preeclampsia is a major cause of maternal and perinatal mortality and morbidity worldwide and responsible for $24 \%$ of all maternal deaths in India. ${ }^{1,2}$

There are various tests which can predict and assess high risk pregnancy like- Non Stress Test (NST), Contraction Stress Test (CST), Biophysical Profile (BPP), modified BPP and Doppler velocimetry. Various authors ${ }^{3}$ compared the efficiency of NST, BPP and abnormal Doppler findings in predicting adverse perinatal outcome in high risk pregnancies in search of a better tool for perinatal outcome. NST is a primary foetal surveillance tool. It is simple, non-invasive, and inexpensive; and has no contraindications. In NST continuous electronic monitoring of bfoetal heart rate (F.H.R.) along with recording of fetal movement is undertaken. NST utilizes the observation that the occurrence of accelerations of the fetal heart rate in response to fetal movements is a reliable indicator of immediate fetal well being. However an abnormal NST is nonspecific and warrants further tests. ${ }^{4}$

The recent introduction of color Doppler flow imaging offers new insight in to the dynamic studies of blood flow and provides the first opportunity for repetitive non-invasive hemodynamic monitoring in pregnancy. ${ }^{2}$ The use of Doppler ultrasound for evaluation of the fetal wellbeing is based on the physical principal of change in frequency of the sound wave when it is reflected by blood flow in fetal vessels. It aims to evaluate blood flow in the vessels supplying the placenta and the fetus. The blood vessels most often used for evaluation in pregnancy are umbilical and middle cerebral arteries in the fetus. Significant Doppler changes occur with reduction in fetal growth at a time when other fetal well being tests are still normal. ${ }^{5}$ Umbilical artery Doppler is the most commonly performed Doppler study for the assessment of the fetal well-being. It is considered necessary in pregnancy complicated by IUGR, oligohydramnios, twin-twin transfusion syndrome and discordant twins.

A decrease in the umbilical artery end diastolic velocity and increase in the Resistance Index or Pulsatility Index can be witnessed once $30 \%$ or more of the placental vasculature is abnormal. ${ }^{6}$ Once $60-70 \%$ of the vasculature is affected, absent or reversed end diastolic flow results. ${ }^{7}$ Reversed end diastolic flow is an ominous finding and is associated with a high mortality rate within 1-7 days if fetus is left in utero. ${ }^{8}$ Doppler findings improve perinatal outcome in high risk pregnancies, reduces antenatal admissions, induction of labour and caesarean section for fetal distress. ${ }^{9}$ Middle cerebral artery (MCA) Doppler velocimetry is considered necessary for evaluation of suspected fetal anaemia in conditions such as isoimmunization and parvovirus B-19 infection. ${ }^{10}$

High risk pregnancies increase the maternal and fetal morbidity and mortality; and there is a need for appropriate investigation which can diagnose it early and predicts the morbidity and mortality. This study was undertaken to determine the etiology of high risk pregnancy and the use of both NST and Doppler velocimetry for antepartum surveillance; and compare the results in terms of perinatal outcome.

\section{METHODS}

It was a prospective cross-sectional hospital based study conducted at Central Referral Hospital (CRH) which is a teaching hospital of Sikkim Manipal Institute of Medical Sciences (SMIMS). The study was conducted over a period of eighteen months between November 2012 and April 2014. One hundred consecutive cases of high risk pregnancies were enrolled into the study and investigated with NST (non-stress test) and Doppler velocimetry and results were correlated with perinatal outcome. In all cases, accurate gestational age was established from detailed menstrual history and ultrasonographic evidence of gestational age. Detailed examination, history and investigation were undertaken in each patient. In these patients antenatal surveillance was done by Doppler and NST and results of these tests were correlated with perinatal outcome.

Inclusion criteria were patients with singleton pregnancy beyond 34 weeks having one or more high risk factors e.g. Post date ( $>40$ weeks gestation), Decreased fetal movement, Gestational diabetes or overt diabetes, Chronic hypertension or pregnancy induced hypertension, Fetal growth restriction, Antepartum hemorrhage, Oligohydramnios, Polyhydramnios, Impaired renal function, Maternal heart disease, Rh-negative pregnancy, anaemia, Bad obstetric history and other medical disorders including thyroid disorder, collagen vascular disease, anti-phospholipid antibody syndrome. Pregnant women below 34 weeks, multiple pregnancy and women with no risk factors were excluded from the study.

\section{Methods for Doppler velocimetry}

A colour Doppler ultrasound apparatus (LOGIQ 5 P-GE series, GE Healthcare, USA) equipped with $3.5 \mathrm{MHz}$ transducer was used for Doppler study. All the examinations were made with the patient lying in semi recumbent position with a lateral tilt. Doppler transducer was placed on the abdominal wall over the uterus and carefully manipulated till Doppler signals appropriate for that particular vessel were identified.

Values measured were defined as: 


\section{Pulsatility index}

It requires digitalized wave form analysis for calculating the mean of frequencies represented.

PI = (Systolic - End diastolic peak velocity $) /$ Timed average maximum velocity

Doppler study was considered abnormal when any of the following parameters were met:

- Pulsatility index of UA $>95^{\text {th }}$ Percentile for the gestational age.

- Absence or reversal of end diastolic flow in umbilical artery.

- Pulsatility index of MCA $<5^{\text {th }}$ Percentile for the gestational age.

- Abnormal cerebro placental ratio- PI MCA/UA $<1.08$.

\section{Methods for NST}

The patient was laid on bed in 15 degree left lateral tilt in order to minimize aortocaval compression. The fetal heart rate transducer was belted on lower abdomen at a site from where the fetal heart is most clearly audible and the toco transducer was belted on the upper abdomen over the uterine fundus. The patient button which is actually a remote event marker was given to the patient so that she can press it as and when she felt any fetal movement and as she does so a mark is printed on the CTG paper. NST was categorized according to FIGO (International Federation of Obstetrics and Gynaecology) guideline.

Based on the Doppler velocimetry and NST results, the study population was divided in to four groups:

- A - Normal NST and Normal Doppler velocimetry

- B - Normal NST and Abnormal Doppler velocimetry

- C - Abnormal NST and Normal Doppler velocimetry

- D - Abnormal NST and abnormal Doppler velocimetry

The management of pregnancy and route of delivery was based on maternal and fetal parameters. This included fetal maturity, NST and Doppler study findings. The perinatal outcome was considered abnormal when any one or combinations of the following were present:

- Perinatal death

- Thick meconium stained liquor

- Apgar $<7$ at 5 minutes

- NICU admissions

- Neonatal seizures within first 24 hours to 48 hours

All results are expressed as mean \pm standard deviation, median or range as appropriate. Discrete variables comparison between different groups was done with Chisquare or Fischer's exact test. Continuous variables in different groups was compared with Student t-test (for 2 groups) or with one-way analysis of variance (for $>2$ groups). For skewed data Mann Whitney test was applied. All statistical tests were two-sided and were performed at significance level of $\mathrm{p}<0.05$. The sensitivity, specificity, positive predictive value and negative predictive value were determined.

\section{RESULTS}

Table 1: Patient profile of the studied women.

\begin{tabular}{|c|c|c|}
\hline Characteristics & Number (n) & Percentage $(\%)$ \\
\hline \multicolumn{3}{|l|}{ Age (in years) } \\
\hline $20-25$ & 25 & $25 \%$ \\
\hline $26-30$ & 41 & $41 \%$ \\
\hline $31-35$ & 25 & $25 \%$ \\
\hline $36-39$ & 9 & $9 \%$ \\
\hline \multicolumn{3}{|l|}{ Parity } \\
\hline Primigravida & 74 & $74 \%$ \\
\hline Multigravida & 26 & $26 \%$ \\
\hline \multicolumn{3}{|c|}{ Gestational age (weeks) } \\
\hline $34-36$ & 14 & $14 \%$ \\
\hline $37-39$ & 79 & $79 \%$ \\
\hline $40-41$ & 7 & $7 \%$ \\
\hline \multicolumn{3}{|l|}{ High risk factors } \\
\hline $\begin{array}{l}\text { Pregnancy induced } \\
\text { hypertension }(\mathrm{PIH})\end{array}$ & 63 & $63 \%$ \\
\hline Oligohydramnios & 11 & $11 \%$ \\
\hline $\begin{array}{l}\text { Gestational Diabetes } \\
\text { Mellitus (GDM) }\end{array}$ & 10 & $10 \%$ \\
\hline Post dated pregnancy & 7 & $7 \%$ \\
\hline Rh negative & 5 & $5 \%$ \\
\hline $\begin{array}{l}\text { Intrauterine growth } \\
\text { retardation }\end{array}$ & 4 & $4 \%$ \\
\hline \multicolumn{3}{|l|}{ Test parameter report } \\
\hline Normal NST & 93 & $93 \%$ \\
\hline Abnormal NST & 7 & $7 \%$ \\
\hline Normal Doppler & 93 & $93 \%$ \\
\hline Abnormal Doppler & 7 & $7 \%$ \\
\hline \multicolumn{3}{|c|}{ Group based on test result } \\
\hline $\begin{array}{l}\text { Group A-NST-Normal } \\
\text { Doppler-Normal }\end{array}$ & 88 & $88 \%$ \\
\hline $\begin{array}{l}\text { Group B-NST-Normal } \\
\text { Doppler-Abnormal }\end{array}$ & 5 & $5 \%$ \\
\hline $\begin{array}{l}\text { Group C-NST- } \\
\text { Abnormal Doppler- } \\
\text { Normal }\end{array}$ & 5 & $5 \%$ \\
\hline $\begin{array}{l}\text { Group D-NST- } \\
\text { Abnormal Doppler- } \\
\text { Abnormal }\end{array}$ & 2 & $2 \%$ \\
\hline \multicolumn{3}{|l|}{ Mode of delivery } \\
\hline Elective LSCS & 52 & $52 \%$ \\
\hline LSCS for fetal distress & 16 & $16 \%$ \\
\hline $\begin{array}{l}\text { Normal vaginal } \\
\text { delivery }\end{array}$ & 32 & $32 \%$ \\
\hline
\end{tabular}

NVD - Normal Vaginal Delivery, LSCS - Lower segment cesarean section, NST - nonstress test. 
A total of hundred consecutive women were studied. The age of the patients in this study ranged from 20 to 39 years of which majority belonged to the age group of 2630 years, with a mean age of $28.68 \pm 4.89$ years. The primigravida comprised of two-third of all the patients while a similar proportion of patient were 37-39 weeks of gestation. Maximum (63\%) patients belonged to pregnancy induced hypertension (PIH) group, $11 \%$ had oligohydramnios, $10 \%$ had gestational diabetes mellitus (GDM), and seven patients had post-dated pregnancy. Table 1 represents the patient profiles of the studied women. All cases were divided into four groups based on NST and Doppler velocimetry of umbilical artery and middle cerebral artery. Sixty eight $(68 \%)$ of high risk pregnant patients underwent lower segment caesarean section (LSCS) and 32\% patients had normal vaginal.

Table 2: Perinatal outcome in four groups.

\begin{tabular}{|c|c|c|c|c|c|}
\hline \multirow{2}{*}{\multicolumn{2}{|c|}{ Perinatal outcome }} & Group A & Group B & Group C & Group D \\
\hline & & $(\mathrm{n}=88)$ & $(n=5)$ & $(\mathrm{n}=5)$ & $(n=2)$ \\
\hline \multirow{3}{*}{ Neonatal outcome } & Healthy & 86 & 2 & 5 & 1 \\
\hline & IUGR & 2 & 1 & 0 & 0 \\
\hline & Neonatal death & 0 & 2 & 0 & 1 \\
\hline \multirow{2}{*}{ Liqour colour } & Clear & 85 & 1 & 5 & 0 \\
\hline & Meconium-stained & 3 & 4 & 0 & 2 \\
\hline \multirow{2}{*}{ APGAR score at 5 minute } & $\geq 7$ at $5 \mathrm{~min}$ & 87 & 0 & 5 & 0 \\
\hline & $<7$ at $5 \mathrm{~min}$ & 1 & 5 & 0 & 2 \\
\hline \multirow{2}{*}{ NICU admissions } & No admissions & 84 & 0 & 5 & 1 \\
\hline & Admissions & 4 & 5 & 0 & 1 \\
\hline Seizures & - & 0 & 0 & 0 & 0 \\
\hline $\begin{array}{l}\text { Overall neonatal } \\
\text { complications }\end{array}$ & - & 9 & 5 & 0 & 2 \\
\hline
\end{tabular}

IUGR - intrauterine growth retardation, NICU - neonatal intensive care unit.

Table 3: Prediction value of abnormal and normal test.

\begin{tabular}{|c|c|c|c|}
\hline $\begin{array}{l}\text { Test } \\
\text { result }\end{array}$ & $\begin{array}{l}\text { No perinatal } \\
\text { complications }\end{array}$ & $\begin{array}{l}\text { Perinatal } \\
\text { complications }\end{array}$ & $\begin{array}{l}P \\
\text { value }\end{array}$ \\
\hline \multicolumn{4}{|c|}{ Abnormal test } \\
\hline $\begin{array}{l}\text { Abnormal } \\
\text { NST }\end{array}$ & 5 & 2 & \multirow[t]{2}{*}{0.021} \\
\hline $\begin{array}{l}\text { Abnormal } \\
\text { Doppler }\end{array}$ & 0 & 7 & \\
\hline \multicolumn{4}{|c|}{ Normal test } \\
\hline $\begin{array}{l}\text { Normal } \\
\text { NST }\end{array}$ & 79 & 14 & \multirow[t]{2}{*}{0.37} \\
\hline $\begin{array}{l}\text { Normal } \\
\text { Doppler }\end{array}$ & 84 & 9 & \\
\hline
\end{tabular}

\section{Perinatal outcome}

Maximum number of high risk pregnant patients belonged to Group A with both normal NST and normal Doppler velocimetry. In Group A, out of total 88 patients 9 had fetal compromise. In Group B, out of 5 patients all had fetal compromise. In Group C, out of 5 patients none had fetal compromise. In Group D, both two patients in this group had fetal compromise. it was found that highest percentage of neonatal complications occurred in groups B and D with abnormal Doppler studies (Table 2). Abnormal Doppler waveform had more adverse perinatal outcome when compared with NST. In group B with total five patients; all newborns had NICU admissions with 2 neonatal deaths. In group $\mathrm{D}$, one had neonatal deaths. No newborn had seizures till discharge.

\section{Correlation between NST, Doppler parameters and perinatal outcome}

NST was normal in 93 high risk pregnant patients and 14 newborns had perinatal complications, and 79 newborns had good perinatal outcome. Seven high risk pregnant patients had abnormal NST, out of which two newborns had perinatal complications and 5 newborns had no perinatal complications. Thus, NST gave correct prediction in 81 cases and in 19 cases wrong prediction of perinatal outcome was given. Perinatal outcome was correctly predicted by Doppler velocimetry in 91 cases and perinatal outcome was not predicted correctly in 9 cases. However, it was not statistically significant on comparing with NST $(\mathrm{p}=0.06)$. Ninety three high risk pregnant patients had normal Doppler velocimetry waveform, out of which 84 newborns had no perinatal complications; however 9 newborns had perinatal complications. Doppler velocimetry was abnormal in 7 high risk pregnant patients and all 7 newborns had perinatal complications (Table 3). 
Doppler velocimetry was able to predict perinatal outcome correctly in 91 cases. Doppler velocimetry was significantly better in predicting the bad perinatal outcome than the NST $(\mathrm{p}=0.021)$. However, statistical significance was not reached in predicting the good perinatal outcome when NST and Doppler velocimetry was compared (Table 3). When NST was compared for different types of perinatal outcome, it was not able to differentiate between the neonatal complications. It did not reach to statistical significance in any of the perinatal outcome (Table 4).

Table 4: NST correlation with perinatal outcome.

\begin{tabular}{|c|c|c|c|c|}
\hline NST & & Normal & Abnormal & P value \\
\hline \multirow{3}{*}{ Condition at discharge } & Healthy & 88 & 6 & \multirow{3}{*}{0.361} \\
\hline & IUGR & 3 & 0 & \\
\hline & Death & 2 & 1 & \\
\hline \multirow{2}{*}{ NICU admission } & No admission & 84 & 6 & \multirow{2}{*}{1.000} \\
\hline & Admission & 9 & 1 & \\
\hline \multirow{2}{*}{ Liqour colour } & Clear & 86 & 5 & \multirow[b]{2}{*}{0.233} \\
\hline & Meconium- stained & 7 & 2 & \\
\hline \multirow{2}{*}{ APGAR category } & $<7$ at 5 minute & 6 & 2 & \multirow{2}{*}{0.174} \\
\hline & $\geq 7$ at 5 minute & 87 & 5 & \\
\hline \multirow{2}{*}{ Overall neonatal complications } & Yes & 14 & 2 & \multirow{2}{*}{0.311} \\
\hline & No & 79 & 5 & \\
\hline
\end{tabular}

NST - non stress test, IUGR - intrauterine growth retardation, NICU - neonatal intensive care unit.

Table 5: Doppler velocimetry and correlation with perinatal outcome.

\begin{tabular}{|c|c|c|c|c|}
\hline NST & & Normal & Abnormal & P value \\
\hline \multirow{3}{*}{ Condition at discharge } & Healthy & 91 & 3 & \multirow{3}{*}{0.001} \\
\hline & IUGR & 2 & 1 & \\
\hline & Death & 0 & 3 & \\
\hline \multirow{2}{*}{ NICU admission } & No admission & 89 & 1 & \multirow{2}{*}{0.001} \\
\hline & Admission & 4 & 6 & \\
\hline \multirow{2}{*}{ Liqour colour } & Clear & 90 & 1 & \multirow{2}{*}{0.001} \\
\hline & Meconium- stained & 3 & 6 & \\
\hline \multirow{2}{*}{ APGAR category } & $<7$ at 5 minute & 1 & 7 & \multirow{2}{*}{0.001} \\
\hline & $\geq 7$ at 5 minute & 92 & 0 & \\
\hline \multirow{2}{*}{ Overall neonatal complications } & Yes & 9 & 7 & \multirow{2}{*}{0.001} \\
\hline & No & 84 & 0 & \\
\hline
\end{tabular}

NST - non stress test, IUGR - intrauterine growth retardation, NICU - neonatal intensive care unit.

Table 6: Pulsatility index (PI) of MCA/UA (Cerebroplacental Ratio) correlation with perinatal outcome.

\begin{tabular}{|c|c|c|c|c|}
\hline PI of MCA/UA & & $\geq 1.08$ & $<1.08$ & P value \\
\hline \multirow{3}{*}{ Condition at discharge } & Healthy & 91 & 3 & \multirow{3}{*}{0.001} \\
\hline & IUGR & 2 & 1 & \\
\hline & Death & 0 & 3 & \\
\hline \multirow{2}{*}{ NICU admission } & No admission & 89 & 1 & \multirow{2}{*}{0.001} \\
\hline & Admission & 4 & 6 & \\
\hline \multirow{2}{*}{ Liqour colour } & Clear & 90 & 1 & \multirow{2}{*}{0.001} \\
\hline & Meconium- stained & 3 & 6 & \\
\hline \multirow{2}{*}{ APGAR category } & $<7$ at 5 minute & 1 & 7 & \multirow{2}{*}{0.001} \\
\hline & $\geq 7$ at 5 minute & 92 & 0 & \\
\hline \multirow{2}{*}{ Overall neonatal complications } & Yes & 9 & 7 & \multirow{2}{*}{0.001} \\
\hline & No & 84 & 0 & \\
\hline
\end{tabular}

PI - Pulsatility index, MCA - middle cerebral artery, UA - umbilical artery, IUGR - intrauterine growth retardation, NICU - neonatal intensive care unit.

Ninety four newborns were healthy at discharge, out of these 91 newborns had normal Doppler studies and 3 newborns had abnormal studies. Three newborns had IUGR at birth out of which Doppler velocimetry was 
abnormal in one newborn. Three newborns died and all had abnormal Doppler velocimetry. NICU admissions were required for 10 newborns, out of these 6 newborns had abnormal Doppler studies. APGAR score $<7$ at 5 minute was found in 8 newborns, out of these 7 had abnormal Doppler velocimetry. Overall neonatal complications were present in 16 newborns and out of these Doppler velocimetry was abnormal in 7 newborns. Doppler studies were significantly abnormal in patients with high risk pregnancy with perinatal complications as compared to the patients with high risk pregnancy without perinatal complications (Table 5).

Table 7: Efficacy of Doppler velocimetry and NST in predicting fetal compromise.

\begin{tabular}{|lllll|}
\hline Test & Sensitivity & Specificity & $\begin{array}{l}\text { Positive predictive } \\
\text { value }\end{array}$ & $\begin{array}{l}\text { Negative predictive } \\
\text { value }\end{array}$ \\
\hline Doppler & 43 & 100 & 100 & 90 \\
\hline Cerebroplacental ratio & 43 & 100 & 100 & 90 \\
\hline NST & 12 & 94 & 28 & 84 \\
\hline
\end{tabular}

NST - non stress test, Doppler - Doppler velocimetry

Three patients had absent/reverse end diastolic flow (ADF/RDF). Out of 3 newborns, 2 died and another 1 was healthy at discharge. All 3 newborns required NICU admissions, had meconium stained liquor and APGAR score was $<7$ at 5 minute. ADF/RDF on Doppler studies was significantly abnormal in patients with high risk pregnancy with perinatal complications as compared to the patients with high risk pregnancy without perinatal complications $(\mathrm{P}<0.001)$.

Seven patients had Cerebroplacental ratio <1.08 i.e. abnormal Doppler parameter. All seven newborns had perinatal complications including 3 neonatal deaths. All 7 newborns had Apgar score $<7$ at 5 minute, 6 newborns required admission in NICU and 6 newborn had meconium stained liquor. Cerebroplacental ratio was significantly abnormal in patients with high risk pregnancy with perinatal complications as compared to the patients with high risk pregnancy without perinatal complications (Table 6). Mean value of PI of MCA and PI of UA were $1.49 \pm 0.34(0.89-2.58)$ and $0.90 \pm 0.20$ $(0.49-1.52)$, respectively. The $5^{\text {th }}, 50^{\text {th }}$, and $95^{\text {th }}$ percentiles of PI of MCA were 1.04, 1.40 and 2.19, respectively and the same for PI of UA were $0.65,0.90$ and 1.29 respectively.

Diagnostic performance of cerebroplacental ratio in predicting fetal outcome was calculated. Sensitivity and Specificity was calculated as $43 \%$ and $100 \%$ respectively. Positive predictive value and negative predictive value came out to be $100 \%$ and $90 \%$, respectively (Table 7).

Sensitivity and specificity of Doppler velocimetry was $43 \%$ and $100 \%$, respectively, whereas, sensitivity and specificity of NST was $12 \%$ and $94 \%$, respectively. Positive predictive value of Doppler velocimetry and NST was $100 \%$ and $28 \%$, respectively. Negative predictive value of Doppler velocimetry and NST was $90 \%$ and $84 \%$, respectively (Table 7).

\section{DISCUSSION}

An analysis of the distribution of surveillance indications showed that the women with the most compromised fetuses such as severe preeclampsia and severe preeclampsia with IUGR belonged to group D, which required a delivery at an earlier gestational age than in Group A which had a greater proportion of mild pre-eclamptic women who continued till term. Considering the mode of delivery, Group C and Group $\mathrm{D}$ had the majority of operative deliveries for suspected fetal compromise.

In present study, the highest percentage of perinatal complications and perinatal deaths were seen in groups with abnormal test results of NST and velocimetry. All five patients in group B had newborns with perinatal complications, meconium staining present in 4 out of 5 newborns, APGAR $<7$ was present in all 5 newborns, and NICU admissions were seen in all 5 newborns, while in group $\mathrm{D}$, both 2 newborns had meconium staining and APGAR $<7$ with one NICU admission and one perinatal death. Average birth weight was also lower in group B and D with abnormal Doppler waveforms, as compared to group $\mathrm{A}$ and $\mathrm{C}$ with normal Doppler waveforms. Yelikar et $\mathrm{al}^{11}$ showed in their study that maximum number of neonates from Group B and D (where Doppler was abnormal) had meconium staining, APGAR <7, and NICU admissions. Similarly, Group D where both the tests (NST and Doppler) were abnormal had the least mean birth weight and a majority of the perinatal deaths were from Group D, six out of 18 women in that group. Both these group had abnormal Doppler studies. Radhika ${ }^{5}$ et al in their study found that Group A where both test results were normal had majority of cases $(55 \% ; 11 / 20)$ and had the least morbidity. Group D which had the maximum number of cases of combined pre-eclampsia with IUGR $(81.25 \%$; 13/16), had both the test results abnormal, and had the worst perinatal outcome 
Since sensitivity is so low, a normal Doppler and normal NST may not be an indicator for fetal well being. However, when the sensitivity and specificity was calculated in a particular gestational age in the study we observed a significant increase in both sensitivity and specificity. Both sensitivity and specificity of Doppler were more than NST. The positive predictive value of Doppler velocimetry was $100 \%$ while that of NST was only $28 \%$. This signifies that an abnormal Doppler study is more accurate in predicting fetal compromise than an abnormal NST. Phelan et $\mathrm{al}^{12}$ studied 3000 NSTs done on 1452 high risk pregnant patients and observed that NST had sensitivity of $64 \%$, specificity of $81 \%$ and false positive rate of $97 \%$ thus showing that NST has low efficacy.

In present study, prediction value of abnormal Doppler velocimetry was significantly higher than NST; and Doppler studies showed a better role in predicting perinatal outcome. A similar comparative study between NST and Doppler waveforms was done by Ott et $\mathrm{al}^{13}$. In that study also Doppler was found to be more efficacious than NST. The fetuses with a normal NST, but abnormal Doppler waveforms had worse outcome than those with an abnormal NST and a normal Doppler study; those for whom both tests were abnormal experienced the worst outcome. This result was similar to present study, which showed the similar findings.

An Indian study ${ }^{5}$, which compared the efficacy of Doppler velocimetry and NST in predicting fetal compromise in utero in cases of severe pre-eclampsia or IUGR, had observed that Doppler waveform abnormalities preceded NST changes with a lead time of 4.14 days. There were 10 perinatal deaths in that study, six of which occurred in the group where both the tests were abnormal. The authors concluded that Doppler study identifies fetal compromise earlier than NST. The lead time helps to plan delivery in preterm compromised pregnancies, resulting in better perinatal survival.

ADF or RDF of umbilical artery correlated with poor perinatal outcome with an increase in the perinatal mortality and morbidity in our study. ADF was associated with $66 \%$ neonatal death in the present study. ADF was associated with $43.7 \%^{14}, 50 \%^{15}$ and $40 \%^{16}$ neonatal deaths, respectively in different studies.

The sensitivity and specificity of cerebro placental ratio was $43 \%$ and $100 \%$ in present study, respectively. However, sensitivity varied from $68 \%{ }^{17}, 83.3 \%{ }^{18}$ and $98 \% 19$ in different studies. The specificity was also variable in different studies, from $66 \%^{19}$ to $100 \%{ }^{18}$. In the study by Bano et $\mathrm{al}^{18} \mathrm{CPR}$ had the highest specificity $(100 \%)$ when compared to other indices in predicting NICU admissions and APGAR $<7$ at 5 minutes. The study showed positive predictive value and negative predictive value as $100 \%$ and $90 \%$, respectively. Fong et $\mathrm{al}^{20}$ in their study found the sensitivity and specificity to be $51.3 \%$ and $80.6 \%$ respectively. In this study positive predictive value and negative value was $48.1 \%$ and $82.5 \%$ respectively. MCA/UA pulsatility index ratio is better in predicting perinatal outcome as it not only incorporates data on the placental status but also on fetal response. Gramellini et al. ${ }^{17}$ found that the cerebroplacental ratio remains constant in the last 10 weeks of pregnancy. They have also shown that it provides a better diagnostic accuracy than umbilical artery and middle cerebral artery pulsatility index considered alone. The results in evaluating the usefulness of umbilical artery and middle cerebral artery Doppler in predicting the adverse perinatal outcome in high risk pregnancy indicate that cerebroplacental ratio is a strong predictor of adverse outcome.

However, there are few limitations of our study, as it was hospital based, had small sample size and there were no control populations. As present study was done in the teaching institution and different scans are done by different radiologists, hence there might be inter observer variations. The aim of future research should be to minimize the risks of fetal morbidity and mortality further by the optimal timing of delivery. There is potential to improve the specificity of fetal surveillance tests, example, better methods of biometry and amniotic fluid volume estimation with three dimensional ultrasound and measurement of subcutaneous tissue. Improved knowledge of fetal physiology can be gained from research of fetal circulation with Doppler studies. Computer analysis of fetal heart rate can increase the specificity of that test and combining both as an integrated test will make optimal use of antenatal fetal surveillance.

\section{CONCLUSION}

PIH was the most common etiology of high risk pregnancy. Doppler velocimetry was better in predicting fetal compromise in comparison to NST in high risk pregnancies. Normal NST and normal Doppler velocimetry were not significantly different in prediction of fetal outcome. Abnormal Doppler value was better in predicting fetal compromise in comparison to abnormal NST. Cerebroplacental ratio was very accurate and was good predictor of adverse perinatal outcome. Both NST and Doppler velocimetry complemented each other in fetal surveillance of high risk pregnancy, although Doppler studies were more efficacious.

Funding: No funding sources

Conflict of interest: None declared

Ethical approval: The study was approved by the Institutional Ethics Committee of SMIMS

\section{REFERENCES}

1. WHO International Collaborative study of Hypertensive disorders of pregnancy. Geographic 
variation in the incidence of Hypertension in pregnancy. Am J Obstet Gynecol. 1988;158:80-3.

2. Confidential enquiry into maternal deaths: Why mothers die? 2000-2002. The sixth report of the confidential enquiries into maternal deaths in UK. London: RCOG Press; 2004. Available from http://bja.oxfordjournals.org/content/94/4/413.full. Accessed on 12 October, 2015.

3. Gonzalez JM, Stamilio DM, Ural S, Macones GA, Odibo AO. Relationship between abnormal fetal testing and adverse perinatal outcomes in intrauterine growth restriction. Am J Obstet Gynecol. 2007;196:48-51.

4. Arias F, Daftary SN, Bhide AG, Editors. Practical guide to High Risk Pregnancy and Delivery. $3^{\text {rd }}$ edition. Elsevier Science Health Science Div. 2009:17-27.

5. Radhika P, Lavanya R. Fetal doppler versus NST as predictors of adverse perinatal outcome in severe preeclampsia and fetal growth restriction. J Obstet Gynecol India. 2006;56:134-8.

6. Giles WB, Trudinger BJ, Baird PJ. Fetal umbilical artery flow velocity wave forms and placental resistance: pathological correlation. $\mathrm{Br} \mathrm{J}$ Obstet Gynaecol. 1985;92:31-8.

7. Morrow RJ, Adamson SL, Bull SB. Effect of placental embolization on the umbilical arterial velocity waveform in fetal sheep. Am J Obstet Gynecol. 1989;161:1055-60.

8. Callen PW. Ultrasonography in Obstetrics and Gynaecology. 4th edition. Philadelphia:W.B.Saunders Company; 2000;214.

9. Mari G, Hanif F, Kruger M, Cosmi E, SantolayaForgas J, Treadwell MC. Middle cerebral artery peak systolic velocity: a new Doppler parameter in the assessment of growth-restricted fetuses. Ultrasound Obstet Gynecol. 2007;29:310-6.

10. American College of Obstetricians and Gynecologists. FAQs: HIV and pregnancy2010.Available http://www.acog.org/-/media/For Patients/faq113.pdf? dmc $=1 \quad \&$ ts $=$ 20140929T 1400559593. Accessed on 12 October 2015.

11. Yelikar KA, Prabhu A, Thakre GG. Role of fetal Doppler and non-stress test in preeclampsia and intrauterine growth restriction. J Obstet Gynaecol India. 2013;63:168-72.
12. Phelan JP. The nonstress test: a review of 3,000 tests. Am J Obstet Gynecol. 1981;139:7-10.

13. Ott WJ. Comparison of the non-stress test with the evaluation of centralization of blood flow for the prediction of neonatal compromise. Ultrasound Obstet Gynecol. 1999;14:38-41

14. Fairlie FM, Moretti M, Walker JJ. Determinants of perinatal outcome in pregnancy-induced hypertension with absence of umbilical artery enddiastolic frequencies. Am J Obstet Gynecol. 1991;164:1084-9.

15. Bhatt CJ, Arora J, Shah MS. Role of color Doppler in pregnancy induced hypertension (a study of 100 cases). Indian J Radiol Imaging. 2003;13:417-20.

16. Malhotra N, Chanana C, Kumar S, Roy K, Sharma JB. Comparison of perinatal outcome of growthrestricted fetuses with normal and abnormal umbilical artery Doppler waveforms. Indian J Med Sci. 2006;60:311-7.

17. Gramellini D, Folli MC, Raboni S, Vadora E, Merialdi A. Cerebral-umbilical Doppler ratio as a predictor of adverse perinatal outcome. Obstet Gynecol. 1992;79:416-20.

18. Bano S, Chaudhary V, Pande S. Color Doppler evaluation of cerebral-umbilical pulsatility ratio and its usefulness in the diagnosis of intrauterine growth retardation and prediction of adverse perinatal outcome. Indian J Radiol Imaging. 2010;20:20-5.

19. Shahinaj R, Manoku N, Kroi E, Tasha I. The value of the middle cerebral to umbilical artery Doppler ratio in the prediction of neonatal outcome in patient with preeclampsia and gestational hypertension. J Prenat Med. 2010;4:17-21.

20. Fong KW, Ohlsson A, Hannah ME, Grisaru S, Kingdom J, Cohen H, et al. Prediction of perinatal outcome in fetuses suspected to have intrauterine growth restriction: Doppler US study of fetal cerebral, renal, and umbilical arteries. Radiology. 1999;213:681-9.

Cite this article as: Choudhury N, Sharma BK, Kanungo BK, Yadav R, Rahman H. Assessment of Doppler velocimetry versus nonstress test in antepartum surveillance of high risk pregnancy. Int $\mathbf{J}$ Reprod Contracept Obstet Gynecol 2017;6:663-70. 